\title{
Análisis de ensayos destructivos de una platina de material compuesto en resina poliester isoftática y fibra de vidrio para aplicaciones automotrices.
}

\author{
Analysis of destructive tests of a plate of composite material in \\ isophthalic polyester resin and fiberglass for automotive \\ applications.
}

Edgar Fabián Sánchez Carrión. ${ }^{1}$, Edison Marcelo Castillo Cárdenas. ${ }^{2}$, Víctor David Bravo Morocho. ${ }^{3} \&$ Juan Marcelo Ramos Flores. ${ }^{4}$

\section{Recibido: 23-04-2019 / Revisado: 25-05-2019 /Aceptado: 24-06-2019/ Publicado: 05-07-2019}

\begin{abstract}
:
DOI: https://doi.org/10.33262/cienciadigital.v3i3.648

The present work analyzes a platen in a process of extrusion of material composed of resin and fiberglass, which is a process for obtaining profiles of constant section based on composite materials by means of fiberglass reinforcement and thermostable matrix. . An experimental study was established under tensile and flexural loads to obtain the characteristics of the stage, in which the characteristics of the composite material were obtained. It is important to detail the characteristics to obtain a suitable resulting product to obtain the necessary characteristics in order to compare their characteristics. results with other similar materials.
\end{abstract}

Keywords: Composite Material, Fiber Reinforced Plastic (Frp), Fiberglass, Concurrent Design, Simulation.

\section{Resumen:}

El presente trabajo se analiza una platina en un proceso de extrusión de material compuesto por resina y fibra de vidrio, que es un proceso para la obtención de perfiles de sección

${ }^{1}$ Escuela Superior Politécnica de Chimborazo, Chimborazo Ecuador, esanchez_c@espoch.edu.ec

2 Escuela Superior Politécnica de Chimborazo, Chimborazo Ecuador, edison.castillo@espoch.edu.ec

${ }^{3}$ Escuela Superior Politécnica de Chimborazo, Chimborazo Ecuador, victor.bravo@espoch.edu.ec

${ }^{4}$ Escuela Superior Politécnica de Chimborazo, Chimborazo Ecuador, victor.bravo@espoch.edu.ec 
constante en base a materiales compuestos por medio refuerzo de fibra de vidrio y de matriz termoestable. Se estableció un estudio experimental bajo cargas de tracción como de flexion para obtener las caracterisicas de la platina, en el cual se obtuvo las carcteristicas del material compuesto es importante detallar las carcateristicas para obtener un producto resultante adecuado para obtener las caracteristicas necesarias para asi comparar sus resultados con otros materiales similares.

Palabras claves: Material Compuesto, Fiber Reinforced Plastic (Frp), Fibra De Vidrio, Diseño Concurrente, Simulación.

\section{Introduccion}

Un material compuesto está conformado por dos o más materiales que son diferentes entre sí, para lo cual existen diferentes métodos de fabricación. El proceso de extrusión también llamado pultrusión, de este tipo de materiales de matriz termoestable, se esquematiza en la figura 1 y consiste en que fibras de vidrio pasen por un recipiente que contiene resina, en la cual las fibras se saturan y pasan por un conformador (molde) en el que se da la sección deseada todo este conjunto resina/fibra es tirado por un sistema hidráulico. Cuando la resina que está cargada de rellenos, acelerantes, agentes desmoldantes, pigmentos y aditivos pasa por el molde calefaccionado activando la polimerización de la resina (curado). Las resinas que se utilizan en este proceso de extrusión pueden ser de éster vinilo, epoxica o poliéster, pero aproximadamente el $90 \%$ es poliéster.

Figura 1. Proceso de extrusión (pultrusion) de material compuesto

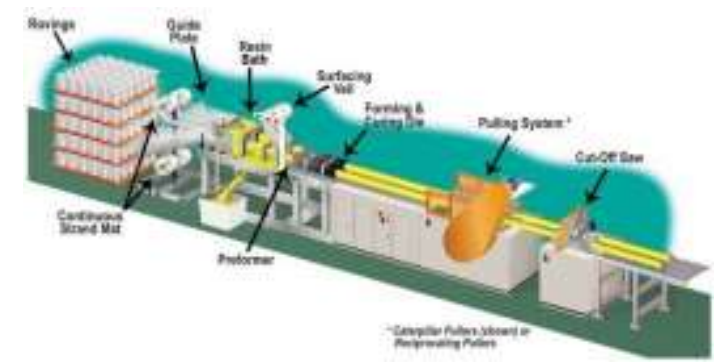

Fuente: Strongwell Corporation.

Al poder crear cualquier tipo de sección transversal de manera constante y de longitud que deseemos es significativa que sus caracteticas mecanicas sean lo suficientemente superiores como para competir con materiales tradicionales en cararteriticas y precio. Dentro de sus ventajas tenemos que su densidad es baja y su baja conductividad térmica y eléctrica y el nulo 
matenimiento; es por ello que es un material importante de caracterizarlo.

\section{Importancia del problema}

En los últimos años se ha experimentado un grande crecimiento en la industria de los materiales compuestos pensando en sus diversos usos y en las diferentes aplicaciones industriales es por ello que tener un conocimiento de sus capacidades mecánicas es de vital importancia para comprender sus usos.

La importancia de este tipo de material se ve en sus múltiples ventajas frente a los materiales ya conocidos, como su propiedad a la corrosión, peso, conductividad eléctrica y térmica, nulo mantenimiento y fácil diseño e instalación. Los campos en los cuales ha estado introduciéndose este material compuesto son las industrias como aeroespaciales, tecnología médica, construcción de vehículos entre otras.

Es por ello que el conocimiento a sus diferentes tipos de ensayos se vuelve importante para poder entender el uso y la importancia de dichos materiales para su uso en los diferentes tipos de industrias.

\section{Metodología}

En el siguiente estudio se centrara en una platina, por lo que es necesario tener un diseño detallado del elemento y la distribución de la resina y de los hilos de fibra de vidrio para tener una repartición adecuada para obtener los mejores características mecánicas. Es importante precisar que estos diseños son detallados y se ajustan a las características de la máquina para poder tener un elemento de alta calidad.

En la platina analizar es necesario precisar que el volumen de resina va a ser del $30 \%$ de área y el $70 \%$ va a ser una mezcla de los rellenos en los cuales tenemos la fibra de vidrio, talco industrial, pigmento entre otros.

\section{Dosificación de la fibra de vidrio}

Es necesario tener en cuenta las características geométricas de la platina para que el producto final obtenga una estabilidad dimensional correcta, la distribución de la fibra dentro del perfil define que en la máquina ingrese correctamente y mantener la sección constante. La cantidad de relleno que ingresamos esta defina por la ecuación 1 . 
Figura 2. Dimensiones del perfil pultruido

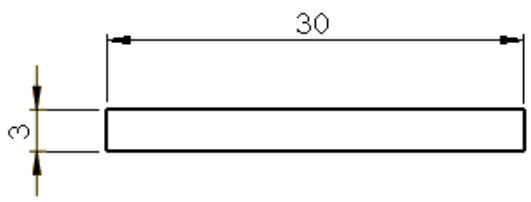

Fuente: Grupo de investigación

En la ecuación 1 realizamos el cálculo del área del perfil a diseñar para poder tener una relación del área a cubrir el material compuesto.

$$
\begin{gathered}
A_{\text {total }}=\text { base } \times \text { altura } \\
A_{\text {total }}=30 \times 3 \\
\text { Area }_{\text {total }}=90 \mathrm{~mm}^{2}
\end{gathered}
$$

El estándar internacional para la fabricación de este tipo de material nos dicta que la matriz en la que va a ser fabrica como longitud máxima tendrá un metro de longitud; la cual nos sirve para obtener el volumen al momento de la fabricación de la platina con la ecuación 2.

Volumen $_{\text {total }}=l_{\text {molde }} * A_{\text {total }}$

$$
\text { Volumen }_{\text {total }}=90000 \mathrm{~mm}^{3}
$$

En la introducción de este trabajo se tiene la consideración que el volumen total va a estar ocupado por el $70 \%$ por la fibra de vidrio y el restante por la resina y sus aditivos. La fibra utilizada para el proceso es una fibra tipo E (para obtener propiedades deseables) y el rendimiento es 2400 TEX, la resina es de tipo isoftalica con las siguientes características:

\begin{tabular}{|c|c|c|c|}
\hline PROPIEDADES DE LA RESINA & A SIN & ARGA (V & LORES TIPICOS \\
\hline Propiedades & Valor & Unidad & Método ensayo \\
\hline Densidad $\left(20^{\circ} \mathrm{C}\right)$ & 1,21 & $\mathrm{~g} / \mathrm{cm}^{3}$ & DIN 53479 \\
\hline Resistencia a la tracción & 70 & $M P a$ & DIN 53455 \\
\hline Módulo de elasticidad en tracción & 4300 & $M P a$ & DIN 53457 \\
\hline Elongación a la ruptura & 2 & $\%$ & DIN 53455 \\
\hline Resistencia a la flexión & 110 & $M P a$ & DIN 53452 \\
\hline
\end{tabular}
Densidad de $1,21 \mathrm{~g} / \mathrm{cm} 3$, resistencia a la tracción de 70 MP1 y están ensayadas bajo la norma DIN53479; el resto de las propiedades están descritas en la tabla 1.

Tabla 1. Propiedades de la resina isoftalica. 
Módulo de elasticidad en flexión

Temperatura de distorsión por calor (HDT)

$\begin{array}{lll}4300 & M P a & \text { DIN 53 457 } \\ 66 & { }^{\circ} \mathrm{C} & \text { DIN EN ISO 75 } \\ 67 & { }^{\circ} \mathrm{C} & \text { ASTM D 648 }\end{array}$

Fuente: Adaptado datos del fabricante 2018.

Con el supuesto de que el perfil tiene el $100 \%$ de su sección constante llena de resina tenemos la siguiente ecuación que tenemos la masa en peso de la platina estudiada.

$$
\begin{gathered}
\text { Masa }=\text { Volumen } * \text { Densidad } \\
\text { masa }=108.9 \mathrm{~g}
\end{gathered}
$$

Como se considera al inicio el $70 \%$ en peso se tiene:

$$
\begin{aligned}
& \text { masa }_{\text {fibra }}=\text { masa }_{\text {resina }} * \% \text { en peso } \\
& \qquad \text { masa }_{\text {fibra }}=108.9 \mathrm{~g} \times 0.70 \\
& \text { masa }_{\text {fibra }}=76.23 \mathrm{~g}
\end{aligned}
$$

Con estos datos es necesario encontrar el número de hilo para lo cual utilizamos la ecuación 5.

$$
\# \text { hilos }=\frac{\text { masa }_{\text {fibra }}}{T_{E X_{\text {fibra }}}}
$$

$$
\begin{aligned}
\# \text { hilos } & =\frac{76.23 \frac{\mathrm{g}}{\mathrm{m}}}{2.4 \frac{\mathrm{g}}{\mathrm{m}}} \\
\text { \#hilos } & =31.76
\end{aligned}
$$

Con lo cual obtener 32 hilos los cuales van a ser distribuidos en la sección de la platina como se lo indica en la figura 3.

Figura 3. Disposición de hilos dentro de la sección transversal.

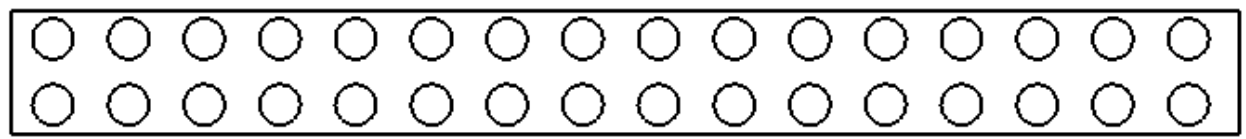

Fuente: Grupo de investigación

Los datos complementarios para obtener nuestra platina de análisis se lo tienen en la tabla 2, la cual tiene provista las velocidades de halado, las temperaturas de curado de la resina y sus aditivos, y el volumen del tanque de resina. 
Tabla 2. Parámetros iniciales de máquina.

\begin{tabular}{llll}
\hline TANQUE & DE & 0.032 & Litro/cm3 \\
RESINA & & & \\
\hline
\end{tabular}

TEMPERATURA CURADO

\begin{tabular}{|c|c|c|c|}
\hline & Zona 1 & Zona 2 & Zona 3 \\
\hline Temperatura $\left({ }^{\circ} \mathbf{C}\right)$ & 70 & 120 & 170 \\
\hline Longitud (mm) & 280 & 220 & 200 \\
\hline $\begin{array}{l}\text { VELOCIDAD } \\
\text { PULLER }\end{array}$ & 0.200 & & $\mathbf{M} / \mathbf{m i n}$ \\
\hline
\end{tabular}

\section{Resultados}

Considerando que el material compuesto es la unión de dos o más elementos diferentes es necesario considerar que el que mayor volumen ocupa es la fibra de vidrio la cuela tenemos que deben ser treinta y dos elementos por lo que la disposición dentro de la sección transversal debe disponerse de tal manera que se cubra la mayoría de la misma para no tener intervalos separados. El diseño del pre conformador es necesario que lleve la disposición dispuesta en este trabajo para que se pueda producir los esfuerzos deseados.

Un elemento que debemos cuidar es la saturación de los hilos con resina, es por ello que al mezclar la resina con los otros componentes es necesario que su exotermia sea controlada para lo cual procedemos a la tabla 3 para ver las concentraciones utilizadas y con las cuales fueron realizados en este estudio.

Tabla 3. Parámetros iniciales de resina.

\begin{tabular}{ll}
\hline MEZCLA A & \\
\hline & Peso[g] \\
Resina poliéster isoftalica & 1000 \\
Percadox 16 & $1 \%$ \\
TBPO & $0.50 \%$ \\
TBPB & $0.25 \%$ \\
Talco industrial & $20 \%$ \\
Alúmina & $1 \%$ \\
\hline
\end{tabular}

Fuente: Grupo de investigación 
Después de realizar las pruebas de mezcla y tener controlada la exotermia se tiene lista la máquina para producir la platina en la cual se va a ensayar. Es importante respetar los parámetros para obtener el perfil con estabilidad dimensional y las características necesarias.

Figura 4. Platina obtenida por la máquina.

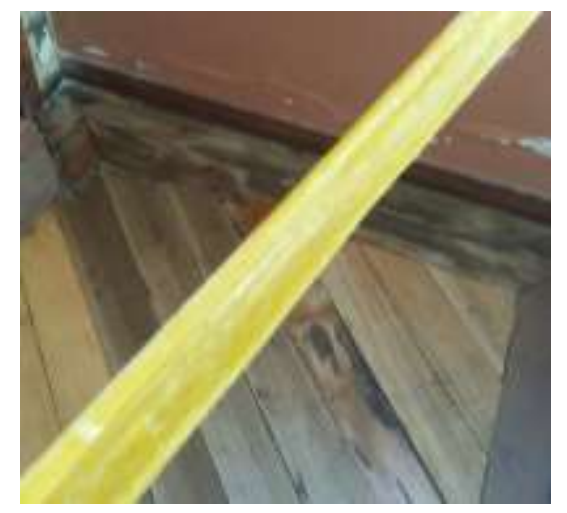

Fuente: Grupo de investigación

Con lo realizado en este estudio nos podemos basar en la regla de las mezclas que se presenta en la ecuación 6 para poder obtener las características con lo cual vamos a analizar de forma longitudinal en dirección al sentido de las fibras. Lo primero que analizamos es la resistencia a la tracción.

$$
\begin{gathered}
E_{c}=E_{m} V_{m}+E_{p} V_{p} \\
(6) \\
E_{c}=(70 M P a) 30 \%+(3.6 G P a) 70 \% \\
E_{c}=2.541 \mathrm{GPa}
\end{gathered}
$$

Con la misma ecuación calculamos el módulo de elasticidad para obtener el equivalente en el material compuesto en forma de platina.

$$
\begin{gathered}
E_{c}=E_{m} V_{m}+E_{p} V_{p} \\
E_{c}=52.04 \mathrm{MPa}
\end{gathered}
$$


Cabe recalcar que la platina ensayada va a ser probada bajo las normas ASTM D3039 que nos sirve para Métodos de ensayo para determinar las propiedades de materiales compuestos de matriz polimérica reforzado con fibras de alto módulo y la ASTM D 7264 las cual Métodos de ensayo para determinar la rigidez a flexión y las propiedades mecánicas de compuestos de matriz polimérica.

\section{Discusión}

La platina ensayada en este elemento es necesario saber que estamos tratando de materiales anisotropicos es decir que en cada uno de sus ejes es diferente el tipo de resistencia que brinda, lo que es evidente en por la disposición de las fibras de vidrio en el material compuesto. En la platina estudiada es necesario entender que los ensayos ASTM D 3039 va a ser realizado en el en dirección de las fibras de vidrio y en el ensayo ASTM D 7264 va a ser ensayado en su eje transversal por lo que la regla de las mezclas debe considerar los ejes.

En la figura 5 podemos observar la disposición de la fibras en el eje a ensayarse por lo cual es donde la tensión está directamente ensayada en los hilos de fibra de vidrio es por ello que se tenía en el preconformador la disposición para que se encuentre bien distribuida en el perfil.

Figura 5. Direccionamiento de hilos dentro de la platina.

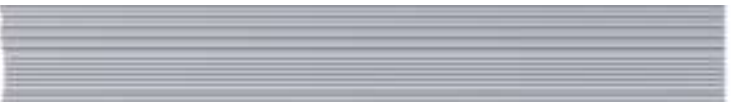

Fuente: Grupo de investigación

Los valores referenciados que admite EPTA (the European Pultrusion Technology Asocciation) se ingresan en la figura la cual nos orientara en los valores obtenidos por nuestra platina.

Figura 6. Propiedades de materiales compuestos según la EPTA

\begin{tabular}{|c|c|c|}
\hline Cemuly & & $165-2.150 \mathrm{gm}$ \\
\hline Remar nompln & bontusua & 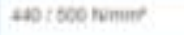 \\
\hline Necura anmegh & tranounas & 200 r 306 Nimer \\
\hline Rescrs imoana of wusachy & engluasin: & m1700/25000 $\mathrm{kmm}$ \\
\hline 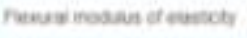 & bewsenas & c4000 yo00 Niment \\
\hline Cambreasen ntryp: & framantes & $240 / 300 \mathrm{Nimm}$ \\
\hline Traie weyh & enchatu: & cos i soe Nume? \\
\hline Trues ituren & trasowna & as 1200 Nemur \\
\hline Everuaten at beas & eonuant & $2.2 \mathrm{~s}$ \\
\hline Eonyater at bertas & Anescanises & 2.65 \\
\hline Ceeffremet af empanam & & $185 \times 10 \times 6 n k-4$ \\
\hline Weat icsorvicit & & $2.15 / 2.56$ \\
\hline
\end{tabular}

Fuente: EPTA. 
En la tabla 5, se tiene los valores anteriormente obtenidos de trabajos anteriores y podremos obtener resultados más fiables.

Tabla 4. Propiedades mecánicas del material compuesto.

\begin{tabular}{ll}
\hline & Mpa \\
\hline modulo a flexion & 2.14 \\
esfuerzo a flexion & 46.23 \\
Módulo a Tesión & $11.56 \mathrm{Gpa}$ \\
Esfuerzo & \\
Tensión & 58.28 \\
\hline
\end{tabular}

Tomandolos valores anteriormente calculados y comparándolos con los valores referenciados podemos ingresarlos a un software y calcularemos su carga y sus esfuerzos.

Con los datos que se obtienen del material ensayamos la platina en un simulador y obtenemos los siguientes resultados, en los que se observa en donde el material comieza a fallar dándose su rotura asi como los valores máximos que se requiere y es 188.1 MPa lo cual esta por debajo de los EPTA nos proporciona, pero mayor al esfuerzo en el material que nos da 58.28 $\mathrm{MPa}$. Bajo estas condiciones tenemos que el factor de seguridad es de 2.4 como lo indica la figura 8 .

Figura 7. Platina simulada en condiciones de norma ASTM D 3039

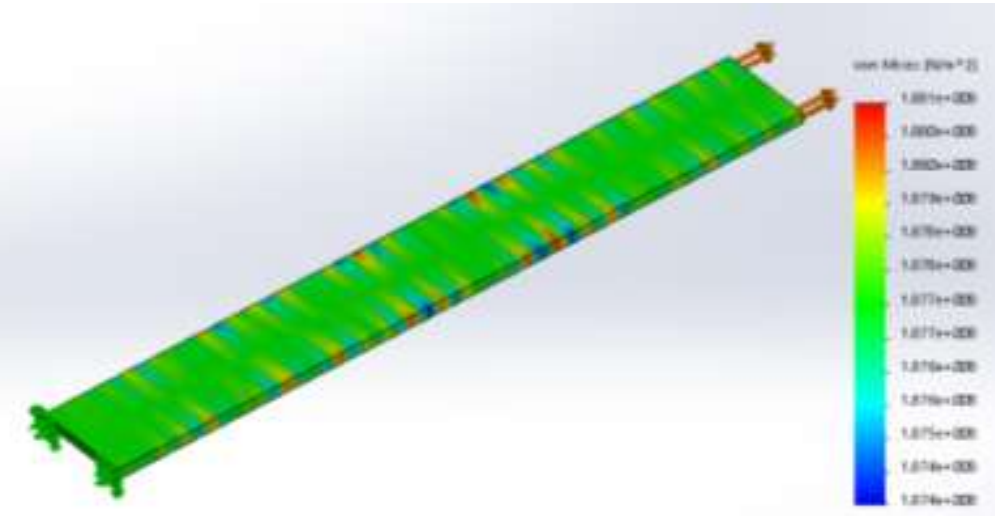

Fuente: Grupo de investigación 
Figura 8. Factor de seguridad de la platina simulada a tracción.

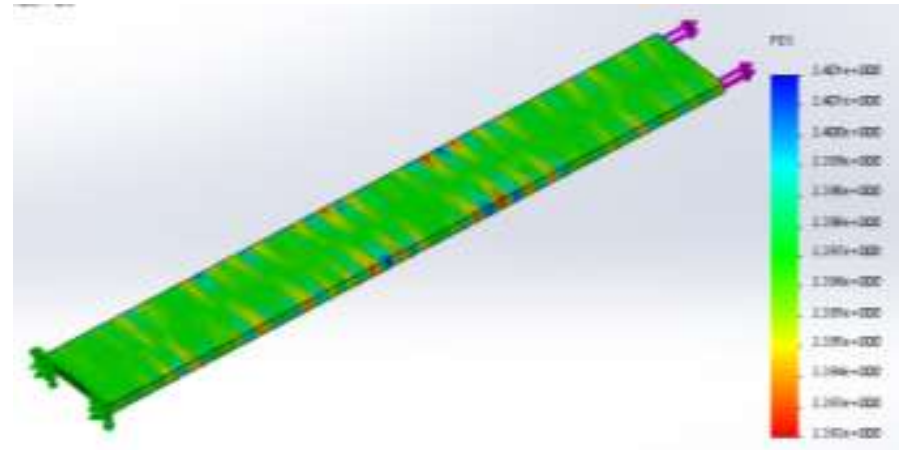

Fuente: Grupo de investigación

Al considerar nuestra platina cen condiciones de flexión tenemos que el resultado es $0.32 \mathrm{MPa}$ lo cual esta debajo de los nos recomienda la EPTA, pero pueden variar por los tipos de refuerzo que introduzcan exteriormente cabe recalcar que este ensayo se lo hizo sin Mat externo. Bajo estas condiciones tenemos que el factor de seguridad es de 8.3 como lo indica la figura 10 .

Figura 9. Platina simulada en condiciones de norma ASTM D 7264
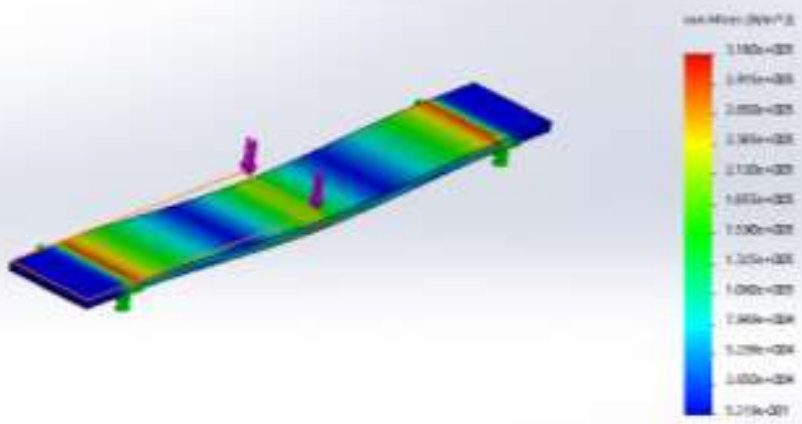

Fuente: Grupo de investigación

Figura 10. Factor de seguridad de la platina simulada a flexión.
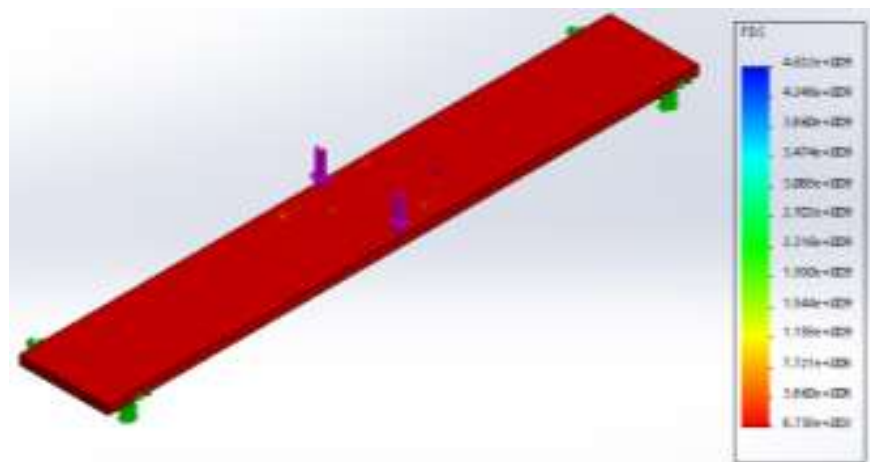

Fuente: Grupo de investigación 
Como se puede observar en la comparación de los valores que existe una diferencia en los valores recomendados por la EPTA, por lo que ellos utilizan procesos de producción de última tecnología así como distintos refuerzo e iniciadores por lo que estos valores son considerablemente altos.

\section{Conclusión}

- Se puede observar que mientras más fibras de vidrio obtenemos mayor resistencia en la tracción es necesario este tipo de cálculos para poder caracterizar el material y posteriormente aplicarlos a la sección de estudio. Las variables de máquina se deben respetar para obtener las características mecánicas deseadas, ya que alguna variación por mínima que sea incide en el resultado final de la misma. Una vez caracterizado el material es necesario llevarlo a un software para poder caracterizarlo y asi poder observar que el porcentaje de error para comparar y tener resultados fiables. Es neceario conocer todos los ensayos que se deben hacer porque estamos tratatanto de materiales compuesto como se lo ha explicado anteriormente son materiales aisotropicos los cuales en un posterior estudio se debe revisar sus resultados en el eje transversal del elementos. Los resultados variarían consderablemente si es que la platina estuviera recubierta con un mat con un tejido entrecruzado para elevar sus propiedas y las características en los ejes transversales se verían beneficiados con este recubrimiento.

\section{Agradecimiento}

Esta investigación no habría sido posible sin el apoyo de la empresa AISA COMPOSITES por lo que agradecemos la disposición por buscar mejoras tecnológicas por el bien de la sociedad y la industria nacional. Además este trabajo investigativo es el fruto del esfuerzo conjunto de conocimientos y experiencias cientificas de los Ingenieros Fabián Sánchez, Marcelo Castillo, David Bravo, Juan Marcelo Ramos Flores. Con estas líneas queremos mostrar nuestro agradecimiento a todas aquellas personas que supieron orientar nuestra trabajo cientifico de una manera correcta.

\section{Referencias Bibliograficas}

Sahuquillo Navarro, O. (2012). Estudio de la degradación de materiales compuestos base poliéster reforzados con fibra de vidrio en medios neutros y alcalinos. Valencia: Tesis doctoral - UPV.

Moschiar S. M.; Reboredo M. M.; Kenny J. M. and Vazquez A. Analysis of pultrusion processing of unsaturaed polyester resin with glass fibers, Polymer Composites, v17, n. 3, p 478, 1996.

Insausti Bello, A. (2007). Estabilidad global de elementos estructurales de polímero reforzado con fribra de vidrio. Donostia: Universidad de Navarra.

Moschiar S. M.;Santiago D.; Lombera G.; Urquiza S. Modelado numerico del proceso de 
pultrusion, Materials Research, Vol 6, No 4, 583-589, 2003.

Bendezú Reyes, J. (2002). Los plasticos reforzdos en fibra de vidrio (PRFV), sus aplicaciones y desarrollo en la industria nacional. Lima- Peru: Universidad nacional Mayor de San Marcos.

Liu X. L.; Crouch I.; Lam Y.; Simulation of heat transfer and cure in pultrusion with a general purpose finite element package, Composites Science and Technology 60 (2000) 857864.

Calabrese L., V. A. (Noviembre, 2002). The effect of a liquid CTBN rubber modifier on the thermo-kinetic parameters of an epoxy resin during a pultrusión process. Composites science and technology, P6.

Guerrero, V., Davila, J., \& Galeas, S. (2011). Nuevos materiales. Aplicaciones estructurales e indsutria. Quito, Ecuador: ImpreFepp.

Raymond W. Meyer, B. (1985). Handbook of pultrusion technology. New York: Chapman and Hall.

Kim D.; Lee W.; Friedrich K. A model for a thermoplastic pultrusion using commingles yarns, Compositees Sciense and Technology 61 (2001) 1065-1077

Guerrero V.; Dávila J.; Galeas S.; Ponton P.; Rosas N.; Sotomayor V.; Valdivieso Nuevos Materiales Aplicaciones estructurles e industriales 2011

Jang, B. Z. (1994). Advanced polymer composites: Principles and applications. ASM International.

Chung, D. (2010). Composite materials, science and applications. New York, EEUU: Springer. 


\section{PARA CITAR EL ARTÍCULO INDEXADO.}

Sánchez Carrión, E., Castillo Cárdenas, E., Bravo Morocho, V., \& Ramos Flores, J. (2019). Análisis de ensayos destructivos de una platina de material compuesto en resina poliester isoftática y fibra de vidrio para aplicaciones automotrices. Ciencia Digital, 3(3), 324-336. https://doi.org/10.33262/cienciadigital.v3i3.648

\section{Ciencia \\ Digital \\ Edtorial}

El artículo que se publica es de exclusiva responsabilidad de los autores y no necesariamente reflejan el pensamiento de la Revista Ciencia Digital.

El artículo queda en propiedad de la revista y, por tanto, su publicación parcial y/o total en otro medio tiene que ser autorizado por el director de la Revista Ciencia Digital.
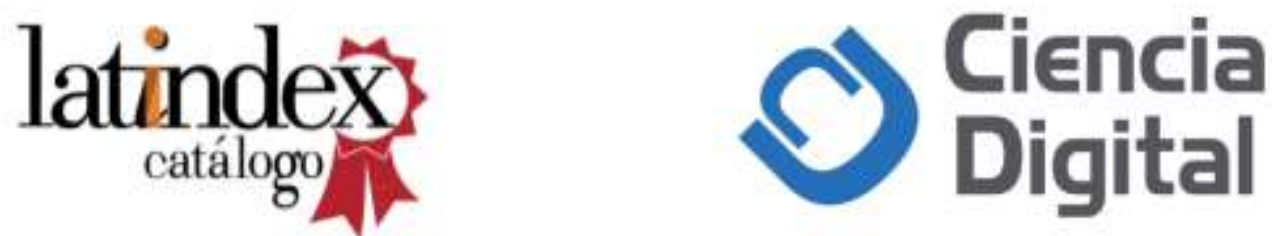